\title{
Nonrelativistic Limit of the Dirac-Schwarzschild Hamiltonian: Gravitational Zitterbewegung and Gravitational Spin-Orbit Coupling
}

\author{
U. D. Jentschura ${ }^{1,2}$ and J. H. Noble ${ }^{1}$ \\ ${ }^{1}$ Department of Physics, Missouri University of Science and Technology, Rolla, Missouri 65409, USA \\ ${ }^{2}$ MTA-DE Particle Physics Research Group, P.O.Box 51, H-4001 Debrecen, Hungary
}

\begin{abstract}
We investigate the nonrelativistic limit of the gravitationally coupled Dirac Equation via a FoldyWouthuysen transformation. The relativistic correction terms have immediate and obvious physical interpretations in terms of a gravitational zitterbewegung, and a gravitational spin-orbit coupling. We find no direct coupling of the spin vector to the gravitational force, which would otherwise violate parity. The particle-antiparticle symmetry described recently by one of us in [Phys.Rev.A 87, 032101 (2013)] is verified on the level of the perturbative corrections accessed by the FoldyWouthuysen transformation. The gravitational corrections to the electromagnetic transition current are calculated.
\end{abstract}

PACS numbers: 11.10.-z, 03.70.+k, 03.65.Pm, 95.85.Ry, 04.25.dg, 95.36.+x, 98.80.-k

\section{INTRODUCTION}

The ever-increasing precision of spectroscopic experiments necessitates the consideration of gravitational effects in relativistic quantum mechanics. General relativity effects already have to be taken into account in the synchronization of clocks in the global positioning system (GPS) satellites, which implies that the satellite-based clocks get ahead of ground-based clocks by about $45 \mu / \mathrm{d}$. For nonrelativistic neutron waves, quantum-mechanical phase shifts due to the gravitational and inertial forces have been measured in Refs. [1 3]. The derivation of the gravitationally coupled Dirac equation has been discussed in textbooks [4-10] as well as the original research literature [11 17]. Recently, it has been argued that a symmetry exists 16] for the gravitationally coupled Dirac equation which implies that particles and antiparticles are both attracted to the gravitational field, and that this symmetry holds to all orders in the velocity of the particles. This symmetry was obtained [16] for a class of static space-time metrics which give rise to a (generalized) Dirac-Schwarzschild equation. We refer to the corresponding Hamiltonian as the Dirac-Schwarzschild Hamiltonian.

However, the result obtained in [16] was not reconciled with other articles from the literature, which investigate the nonrelativistic limit of the quantum dynamics, and, in particular, discuss the conceivable presence [18] of a spin-gravity coupling of the form $\vec{\Sigma} \cdot \vec{g}$, where $\vec{\Sigma}$ is the $(4 \times 4)$-spin matrix, and $\vec{g}$ is the acceleration due to gravity. Specifically, in Ref. [18], a conceivable spin-gravity interaction and the pertinent experimental detection have been investigated. In Ref. [19], via a Foldy-Wouthuysen transformation [20] of the Dirac-Schwarzschild Hamiltonian, a term proportional to $\vec{\Sigma} \cdot \vec{g}$ is obtained in the leading nonrelativistic approximation. It has been discussed in Refs. 21, 22] whether such a term would violate parity. Indeed, it is well known that orbital as well as spin angular momenta constitute pseudo-vectors. Notably, the spin operator $\vec{\Sigma}=\gamma^{5} \gamma^{0} \vec{\gamma}$ transforms under parity as $\vec{\Sigma} \rightarrow \gamma^{0} \vec{\Sigma} \gamma^{0}=\vec{\Sigma}$ and therefore as a pseudovector. By contrast, the gravitational force $\vec{F}_{g}=m \vec{g}$ with $m g=m|\vec{g}|=G m M / r^{2}=m r_{s} /(2 r)$ is a vector. (Here, $m$ and $M$ are the masses of the test particle and planet, respectively, and $r_{s}$ is the Schwarzschild radius; we use natural units with $\hbar=c=\epsilon_{0}=1$ ). One might thus conclude that any term in the Hamiltonian proportional to $\vec{\Sigma} \cdot \vec{g}$ would indeed violate parity symmetry. Apparently, the question of how to physically interpret the leading relativistic corrections in a curved space-time akin to the Schwarzschild geometry still constitutes an open problem [23 26].

The gravitational Dirac Hamiltonian is similar in its mathematical structure to the Dirac-Coulomb Hamiltonian [27 29], and we would a priori expect that the Foldy-Wouthuysen transformation should yield similar terms, but respect the particle-antiparticle symmetry from [16]. The nature of the Foldy-Wouthuysen program is inherently perturbative. In the following, we expand to first order in the gravitational coupling constant, i.e., we only keep terms of first order in the Schwarzschild radius $r_{s}$. The corresponding dimensionless expansion parameter is $\chi=r_{s} / r$, where $r$ measures the distance from the center of the black hole (in the sense of a space-time coordinate). Regarding the momenta and distances, we assume that the Cartesian components $p^{i}$ are of order $\xi m$ and that $r^{i} \sim 1 /(\xi m)$, and expand to order $\chi \xi^{3}$ or to order $\xi^{4}$ (for the gravitationally uncoupled terms). After a rederivation of the Hamiltonian form of the DiracSchwarzschild equation in Sec. II] the Foldy-Wouthuysen transformation is carried out explicitly in Sec. III, while conclusions are reserved for Sec. IV] We again reemphasize the use of natural units throughout the article $\left(\hbar=c=\epsilon_{0}=1\right)$.

\section{FORMALISM}

We use the same conventions as in Refs. [16] and assume that the curved-space Dirac $\gamma$ (overlined) and flat- 
space (tilde) Dirac matrices fulfill the relations

$$
\left\{\bar{\gamma}^{\mu}(x), \bar{\gamma}^{\nu}(x)\right\}=2 \bar{g}^{\mu \nu}(x), \quad\left\{\widetilde{\gamma}^{\mu}, \widetilde{\gamma}^{\nu}\right\}=2 \widetilde{g}^{\mu \nu} .
$$

Here, $\{\cdot, \cdot\}$ is the anticommutator. The curved-space metric is $\bar{g}^{\mu \nu}$ with $\mu, \nu=0,1,2,3$, while the "West-Coast" flat-space metric is $\widetilde{g}^{\mu \nu}=\operatorname{diag}(1,-1,-1,-1)$. Overlining the curved-space Dirac matrices and using the tilde for the flat-space variants avoids a conceivable confusion with the particle physics literature [30 34], where one denotes the flat-space matrices as $\gamma^{\mu}$, and the flat-space metric as $g^{\mu \nu}$, whereas in the literature on general relativity, one usually denotes the curved-space Dirac matrices without a tilde, and uses $g^{\mu \nu}$ for both flat-space as well as curved-space metrics 35 39]. Explicit use of the overlining and the tilde avoids any possible confusion.

The formulation of the gravitationally coupled Dirac equation [4 16] suggests the use of the vierbein formalism, which is particularly straightforward to formulate if the "square root of the metric" can be taken with ease (see Sec. 6 of Ref. [40]). The Dirac action in curved spacetime is given by the invariant integral

$$
S=\int \mathrm{d}^{4} x \sqrt{-\operatorname{det} \bar{g}} \bar{\psi}(x)\left(\frac{\mathrm{i}}{2} \bar{\gamma}^{\rho}(x) \overleftrightarrow{\nabla}_{\rho}-m\right) \psi(x),
$$

where the covariant derivative is $\nabla_{\rho}=\partial_{\rho}-\Gamma_{\rho}$, and

$$
\Gamma_{\rho}=-\frac{\mathrm{i}}{4} \bar{g}_{\mu \alpha}\left(\frac{\partial b_{\nu}{ }^{\beta}}{\partial x^{\rho}} a^{\alpha}{ }_{\beta}-\Gamma_{\nu \rho}^{\alpha}\right) \bar{\sigma}^{\mu \nu} .
$$

is the affine spin-connection matrix. Here, the curvedspace spin matrices are $\bar{\sigma}^{\mu \nu}=\frac{\mathrm{i}}{2}\left[\bar{\gamma}^{\mu}, \bar{\gamma}^{\nu}\right]$. We represent the $\bar{\gamma}^{\nu}$ matrices in terms of the flat-space $\widetilde{\gamma}^{\mu}$,

$$
\begin{aligned}
\bar{\gamma}_{\rho}=b_{\rho}{ }^{\alpha} \widetilde{\gamma}_{\alpha}, & \tilde{\gamma}_{\rho}=a_{\rho}^{\alpha} \bar{\gamma}_{\alpha}, \\
\bar{\gamma}^{\alpha}=a^{\alpha}{ }_{\rho} \widetilde{\gamma}^{\rho}, & \widetilde{\gamma}^{\alpha}=b_{\rho}{ }^{\alpha} \bar{\gamma}^{\rho} .
\end{aligned}
$$

We use the flat-space Dirac matrices in the Dirac representation,

$$
\begin{aligned}
& \widetilde{\gamma}^{0}=\left(\begin{array}{cc}
\mathbb{1}_{2 \times 2} & 0 \\
0 & -\mathbb{1}_{2 \times 2}
\end{array}\right), \quad \widetilde{\gamma}^{1}=\left(\begin{array}{cc}
0 & \sigma^{1} \\
-\sigma^{1} & 0
\end{array}\right), \\
& \widetilde{\gamma}^{2}=\left(\begin{array}{cc}
0 & \sigma^{2} \\
-\sigma^{2} & 0
\end{array}\right), \quad \widetilde{\gamma}^{3}=\left(\begin{array}{cc}
0 & \sigma^{3} \\
-\sigma^{3} & 0
\end{array}\right), \\
& \widetilde{\gamma}^{5}=\mathrm{i} \widetilde{\gamma}^{0} \widetilde{\gamma}^{1} \widetilde{\gamma}^{2} \widetilde{\gamma}^{3}=\left(\begin{array}{cc}
0 & \mathbb{1}_{2 \times 2} \\
\mathbb{1}_{2 \times 2} & 0
\end{array}\right) .
\end{aligned}
$$

The metric is recovered as

$$
\begin{aligned}
& \left\{\bar{\gamma}_{\rho}, \bar{\gamma}_{\sigma}\right\}=b_{\rho}{ }^{\alpha} b_{\sigma}{ }^{\beta}\left\{\widetilde{\gamma}_{\alpha}, \widetilde{\gamma}_{\beta}\right\}=2 \widetilde{g}_{\alpha \beta} b_{\rho}{ }^{\alpha} b_{\sigma}{ }^{\beta}=2 \bar{g}_{\rho \sigma}, \\
& \left\{\bar{\gamma}^{\rho}, \bar{\gamma}^{\sigma}\right\}=a_{\alpha}^{\rho} a_{\beta}^{\sigma}\left\{\widetilde{\gamma}^{\alpha}, \widetilde{\gamma}^{\beta}\right\}=2 \widetilde{g}^{\alpha \beta} a^{\rho}{ }_{\alpha} a^{\sigma}{ }_{\beta}=2 \bar{g}^{\rho \sigma} .
\end{aligned}
$$

The gravitationally coupled Dirac equation, obtained by variation of Eq. (2), is

$$
\left(\mathrm{i} \bar{\gamma}^{\mu} \nabla_{\mu}-m\right) \psi(x)=0 \text {. }
$$

We now specialize to a static space-time metric [41] of a generalized Schwarzschild type,

$$
\bar{g}_{\mu \nu}=\operatorname{diag}\left(w^{2}(r),-v^{2}(r),-v^{2}(r),-v^{2}(r)\right),
$$

where the vierbein coefficients are given as $b_{0}{ }^{\beta}=$ $\delta_{0}{ }^{\beta} w(r), b_{i}{ }^{j}=\delta_{i}{ }^{j} v(r), a^{\alpha}{ }_{0}=\delta^{0}{ }_{\alpha} / w(r)$, and $a_{i}{ }^{j}=$ $\delta_{i}{ }^{j} / v(r)$. The $a$ and $b$ matrices are symmetric in this case, $a^{\mu}{ }_{\nu}=a^{\nu}{ }_{\mu}$ and $b_{\mu}{ }^{\nu}=b_{\nu}{ }^{\mu}$. With these coefficients, using computer algebra [42], it is easy to evaluate all Christoffel symbols and to establish that [16]

$$
\begin{aligned}
\bar{\gamma}^{0} \bar{\gamma}^{\mu} \Gamma_{\mu} & =-\frac{\widetilde{\gamma}^{0} \overrightarrow{\widetilde{\gamma}} \cdot \vec{r}}{r} G(r), \\
G(r) & =\frac{2 v^{\prime}(r) w(r)+v(r) w^{\prime}(r)}{2 v^{2}(r) w^{2}(r)} .
\end{aligned}
$$

The Hamiltonian form of the gravitationally coupled Dirac equation,

$$
\mathrm{i}\left(\bar{\gamma}^{0}\right)^{2} \partial_{t} \psi=\left(\bar{\gamma}^{0} \bar{\gamma}^{j} p^{j}+\mathrm{i} \bar{\gamma}^{0} \bar{\gamma}^{\mu} \Gamma_{\mu}+\bar{\gamma}^{0} m\right) \psi,
$$

translates into $\mathrm{i} \partial_{t} \psi=H \psi$, where $H$ is given by

$$
H=\frac{w}{v} \vec{\alpha} \cdot \vec{p}-\frac{\mathrm{i}}{2 v} \vec{\alpha} \cdot \vec{\nabla} w-\frac{\mathrm{i} w}{v^{2}} \vec{\alpha} \cdot \vec{\nabla} v+\beta m w .
$$

Here, we use the notation $\vec{\alpha}=\widetilde{\gamma}^{0} \vec{\gamma}$ and $\beta=\widetilde{\gamma}^{0}$. We now stretch space according to the scaling

$$
\psi^{\prime}=v^{3 / 2} \psi, \quad H^{\prime}=v^{3 / 2} H v^{-3 / 2} .
$$

This leads to a Hermitian Hamiltonian, which acts on the Hilbert space of square-integrable functions with the scalar product $\langle\phi, \psi\rangle=\int \mathrm{d}^{3} \phi^{*}(\vec{r}) \psi(\vec{r})$. in three-space reads as

$$
H^{\prime}=\frac{1}{2}\{\vec{\alpha} \cdot \vec{p}, \mathcal{F}\}+\beta m w, \quad \mathcal{F}=\frac{w}{v} .
$$

We here confirm the result given in Eq. (14) of Ref. [19]. For the Schwarzschild metric in isotropic coordinates (see Sec. 43 of Chap. 3 of Ref. [41]), we have to first order in the Schwarzschild radius $r_{s}$,

$$
\begin{aligned}
w & =\left(1-\frac{r_{s}}{4 r}\right)\left(1+\frac{r_{s}}{4 r}\right)^{-1}=\frac{4 r-r_{s}}{4 r+r_{s}} \approx 1-\frac{r_{s}}{2 r} \\
v & =\left(1+\frac{r_{s}}{4 r}\right)^{2} \approx 1+\frac{r_{s}}{2 r} \\
\frac{w}{v} & =\frac{16 r^{2}\left(4 r-r_{s}\right)}{\left(4 r+r_{s}\right)^{3}} \approx 1-\frac{r_{s}}{r}
\end{aligned}
$$

The Schwarzschild radius is given as $r_{s}=2 G M$, where $G$ is Newton's gravitational constant, and $M$ is the mass of the planet. So, to first order in $r_{s}$, we have to analyze the Dirac-Schwarzschild Hamiltonian $H_{\mathrm{DS}}$, which is given by

$$
H_{\mathrm{DS}}=\frac{1}{2}\left\{\vec{\alpha} \cdot \vec{p},\left(1-\frac{r_{s}}{r}\right)\right\}+\beta m\left(1-\frac{r_{s}}{2 r}\right) .
$$

We can now carry through the Foldy-Wouthuysen program as usual. 


\section{FOLDY-WOUTHUYSEN TRANSFORMATION}

\section{A. Transformation of the Hamiltonian}

Contrary to widespread belief, the rationale of the Foldy-Wouthuysen transformation 20] actually is rather well-defined [30] and in some sense tied to the Dirac representation of the Dirac matrices given in Eq. (5). One has to (i) identify the odd (in spinor space) part of the Hamiltonian $H$ and denotes this odd part as $\mathcal{O}$. (ii) One then defines the Hermitian operator $S=-\mathrm{i} \beta \mathcal{O} /(2 m)$ and the unitary operator $U=\exp (\mathrm{i} S)$. (iii) The calculation of multiple nested commutators of $U$ and $H$ proceeds until further nested commutators only yield higherorder terms when expressed in terms of defined operational parameters of the expansion. (iv) If there are odd terms left after the first transformation, to the desired order in the expansion, then one employs a second Foldy-Wouthuysen transformation by identifying the odd part of the new Hamiltonian, which resulted from the first Foldy-Wouthuysen step, as $\mathcal{O}^{\prime}$. One does this recursively until all odd parts of the input Hamiltonian are eliminated. In the case of the free Dirac Hamiltonian, it is possible to perform a Foldy-Wouthuysen transformation to all orders in the momenta [30], but one may also choose a perturbative approach (see Appendix A). However, an exact Foldy-Wouthuysen transformation has not been described for more complicated Hamiltonians like the Dirac-Coulomb Hamiltonian 27 29]

$$
H_{\mathrm{DC}}=\vec{\alpha} \cdot \vec{p}+\beta m-\frac{Z \alpha}{r},
$$

where $Z$ is the nuclear charge number and $\alpha$ is the finestructure constant, or for other, nontrivial extensions of the standard free Dirac equation [43]. The DiracSchwarzschild Hamiltonian (15) still is of an intricate nature. A nonperturbative Foldy-Wouthuysen transformation has not been described in the literature for the Dirac-Coulomb Hamiltonian (16), and we can thus conclude that a perturbative approach seems most promising for the Dirac-Schwarzschild Hamiltonian.

For the gravitational correction terms, the expansions below are carried through to to first order in $\xi=r_{s} / r$ and to third order in $\xi=\vec{p} / m$ or $\xi=1 /(m r)$, and we assume the exchanged photons to be soft, i.e., $k \sim \xi^{2} m$. This is the same expansion as for the Lamb shift [30, 44] if we identify $\chi$ with $Z \alpha$, where $Z$ is the nuclear charge number, and $\alpha$ is the fine-structure constant. Terms are calculated up to order $\chi^{4}$ is there is no gravitational interaction, and up to order $\xi \chi^{3}$ if there is a gravitational interaction. Terms of order $\xi^{2}$ (second order in the gravitational interaction) are ignored.

To leading order in $r_{s}$, we have to analyze the Hamiltonian $H_{1}=H_{\mathrm{DS}}$ as given in Eq. (15). For the first FoldyWouthuysen transformation, we therefore have

$$
S_{1}=-\frac{\mathrm{i}}{2 m} \beta \mathcal{O}_{1}, \quad \mathcal{O}_{1}=\frac{1}{2}\left\{\vec{\alpha} \cdot \vec{p},\left(1-\frac{r_{s}}{r}\right)\right\} .
$$

The transformation is calculated as

$$
\begin{aligned}
H_{2} & =\mathrm{e}^{\mathrm{i} S_{1}} H_{1} \mathrm{e}^{-\mathrm{i} S_{1}} \\
& =H_{1}+\mathrm{i}\left[S_{1}, H_{1}\right]+\frac{\mathrm{i}^{2}}{2 !}\left[S_{1},\left[S_{1}, H_{1}\right]\right]+\ldots
\end{aligned}
$$

The result is

$$
\begin{aligned}
H_{2}= & \beta\left(m+\frac{\vec{p}^{2}}{2 m}-\frac{\vec{p}^{4}}{8 m^{3}}\right)-\beta \frac{m r_{s}}{2 r} \\
& +\beta\left(-\frac{3 r_{s}}{8 m}\left\{\vec{p}^{2}, \frac{1}{r}\right\}+\frac{3 \pi r_{s}}{4 m} \delta^{(3)}(\vec{r})+\frac{3 r_{s}}{8 m} \frac{\vec{\Sigma} \cdot \vec{L}}{r^{3}}\right) \\
& -\frac{(\vec{\alpha} \cdot \vec{p})^{3}}{3 m^{2}}+\frac{1}{4}\left\{\frac{r_{s}}{r}, \vec{\alpha} \cdot \vec{p}\right\} .
\end{aligned}
$$

A central ingredient of the Foldy-Wouthuysen transformation is presence of the term $\beta m$ in the initial Hamiltonian and the commutator relation $[\beta \mathcal{O}, \beta m]=-2 m \mathcal{O}$, which holds for any odd (in the spinor space) term $\mathcal{O}$ in the Hamiltonian. Indeed, the first commutator in equation (18) then eliminates the leading odd terms in the initial Hamiltonian $H_{1}$. One might think that this scheme is not applicable to the Hamiltonian (15), because the mass term is multiplied by a factor $\left[1-r_{s} /(2 r)\right]$, but this is not the case: The nature of the Foldy-Wouthuysen transformation is perturbative, and the term $-\beta m r_{s} /(2 r)$ is a perturbative gravitational correction to the mass. The modification of the mass term therefore is of higher order in $\chi$ and does not inhibit the application of the perturbative approach to the Foldy-Wouthuysen transformation.

For the second Foldy-Wouthuysen transformation, we have

$$
S_{2}=-\frac{\mathrm{i}}{2 m} \beta \mathcal{O}_{2}, \quad \mathcal{O}_{2}=-\frac{(\vec{\alpha} \cdot \vec{p})^{3}}{3 m^{2}}+\frac{1}{4}\left\{\frac{r_{s}}{r}, \vec{\alpha} \cdot \vec{p}\right\} .
$$

The transformation is calculated as $H_{\mathrm{FW}}=\mathrm{e}^{\mathrm{i} S_{2}} H_{2} \mathrm{e}^{-\mathrm{i} S_{2}}$. Taking notice of the well-known identity $\vec{p}^{2}\left(\frac{1}{r}\right)=$ $4 \pi \delta^{(3)}(\vec{r})$, the Foldy-Wouthuysen transformation gives the result,

$$
\begin{aligned}
& H_{\mathrm{FW}}=\beta\left(m+\frac{\vec{p}^{2}}{2 m}-\frac{\vec{p}^{4}}{8 m^{3}}\right)-\beta \frac{m r_{s}}{2 r} \\
& +\beta\left(-\frac{3 r_{s}}{8 m}\left\{\vec{p}^{2}, \frac{1}{r}\right\}+\frac{3 \pi r_{s}}{4 m} \delta^{(3)}(\vec{r})+\frac{3 r_{s}}{8 m} \frac{\vec{\Sigma} \cdot \vec{L}}{r^{3}}\right) .
\end{aligned}
$$

The very last term on the right-hand side of Eq. (21) describes the gravitational spin-orbit coupling; it is in agreement with the classical result for the interaction of a spinning classical particle with the gravitational field, which is otherwise known as the geodesic precession or Fokker precession [45 47]. When comparing the spinorbit term with Eq. (26) of Ref. [47], which has a prefactor of $3 / 2$ instead of $3 / 8$, one should take note that the spin operator carries a factor one half $\left(\vec{S}=\frac{1}{2} \vec{\Sigma}\right)$, and there is an additional factor 2 in the definition of 
the Schwarzschild radius. The prefactor $\beta$ in Eq. (21) describes the particle-antiparticle symmetry, which cannot be obtained based on classical considerations [45 47]. The result (21) thus offers a straightforward physical interpretation, as follows: First, we have the usual relativistic corrections to the kinetic energy. The second term on the right-hand side of Eq. (21) is the gravitational potential, duly decorated with a $\beta$ prefactor which ensures that antiparticles are attracted. The third term consists of a kinetic correction to the gravitational coupling, and a Darwin (zitterbewegung) term which is not experimentally relevant because it is located at the center of the planet, i.e., inside the Schwarzschild radius. However, for completeness, we should include this term. Finally, the spin-orbit coupling term commutes with the Dirac angular operator $K=\beta(\vec{\Sigma} \cdot \vec{L}+1)$ and with the total angular momentum (orbital+spin).

Let us conclude the discussion by pointing out a subtlety. One might think that, if the total angular momentum operator commutes with the Hamiltonian, then it should automatically commute with the FoldyWouthuysen transformed Hamiltonian. However, that is not the case. If $A$ is an operator that commutes with the Hamiltonian, $\left[H_{\mathrm{DS}}, A\right]=0$, and $H_{\mathrm{FW}}=U H_{\mathrm{DS}} U^{-1}$, then then the transformed operator $A_{\mathrm{FW}}=U A U^{-1}$ commutes with $H_{\mathrm{FW}}$. This can be seen as follows,

$$
\left[H_{\mathrm{FW}}, A_{\mathrm{FW}}\right]=U\left[H_{\mathrm{DS}}, A\right] U^{-1}=0,
$$

So, if $\vec{J}$ commutes with $H_{\mathrm{DS}}$, then this does not automatically mean that $\vec{J}$ commutes with $H_{\mathrm{FW}}$. Let us recall that the total angular momentum $\vec{J}$ and the Dirac angular operator $K$ are defined as follows,

$$
\vec{J}=\vec{L}+\frac{1}{2} \vec{\Sigma}, \quad K=\beta(\vec{\Sigma} \cdot \vec{L}+1) .
$$

We can establish the following commutator relations,

$$
\begin{aligned}
{\left[H_{\mathrm{DS}}, K\right] } & =0, & & {\left[H_{\mathrm{DS}}, \vec{J}\right]=\overrightarrow{0} . } \\
{\left[H_{\mathrm{FW}}, K\right] } & =0, & & {\left[H_{\mathrm{FW}}, \vec{J}\right]=\overrightarrow{0} . }
\end{aligned}
$$

In view of (22), it is not a triviality to separately establish that both $\vec{J}$ and $K$ commute with $H_{\mathrm{DS}}$ and $H_{\mathrm{FW}}$, individually, but the relations hold. Eigenfunctions of $H_{\mathrm{DS}}$ and of $H_{\mathrm{FW}}$ are eigenstates of $\vec{J}$ and $K$, i.e., the spinor components of the eigenfunction are $\chi_{\varkappa \mu}(\hat{r})$ (upper component) and $\chi_{-\varkappa \mu}(\hat{r})$ (lower component), and they have the same $j=|\varkappa|-1 / 2$.

\section{B. Transformation of the Transition Current}

The main result derived in the current work concerns the Foldy-Wouthuysen Hamiltonian $H_{\mathrm{FW}}$ derived in Eq. (21). However, it is also interesting to investigate the gravitational corrections to the electromagnetic transition current. The transition current operator for the emission of transverse photons in flat space is given by the matrix-valued expression $j^{i}=\alpha^{i} \exp (\mathrm{i} \vec{k} \cdot \vec{r})$; an illustrative discussion can be found in Refs. [30, 44, 48]. The Hermitian adjoint of this operator is obtained by the replacement $\exp (\mathrm{i} \vec{k} \cdot \vec{r}) \rightarrow \exp (-\mathrm{i} \vec{k} \cdot \vec{r})$, i.e., by the replacement of a photon emission by a photon absorption process.

The Dirac-Schwarzschild Hamiltonian (15) is coupled to an external electromagnetic field by the replacement $\vec{p} \rightarrow \vec{p}-e \vec{A}$, where $\vec{A}$ is the vector potential. The interaction Hamiltonian is $H_{\text {int }}=-\vec{j} \cdot \vec{A}$. So, with relativistic gravitational coupling included, the transition current reads as

$$
j^{i}=\frac{1}{2}\left\{1-\frac{r_{s}}{r}, \alpha^{i} \exp (\mathrm{i} \vec{k} \cdot r)\right\}
$$

We now employ the multipole expansion

$$
\alpha^{i} \exp (\mathrm{i} \vec{k} \cdot r) \approx \alpha^{i}+\alpha^{i}(\mathrm{i} \vec{k} \cdot \vec{r})-\frac{1}{2} \alpha^{i}(\vec{k} \cdot r)^{2}
$$

in Eq. (25). A subsequent calculation of the FoldyWouthuysen transformation $j_{\mathrm{FW}}^{i}=U j^{i} U^{-1}$ of the transition current [with $\left.U=\exp \left(\mathrm{i} S_{2}\right) \exp \left(\mathrm{i} S_{1}\right)\right]$ gives the result,

$$
\begin{aligned}
j_{\mathrm{FW}}^{i}= & \frac{p^{i}}{m}-\frac{p^{i} \vec{p}^{2}}{2 m}-\frac{\mathrm{i}}{2 m}(\vec{k} \times \vec{\sigma})^{i}+\frac{1}{2}\left\{\frac{p^{i}}{m},(\mathrm{i} \vec{k} \cdot \vec{r})\right\} \\
& -\frac{1}{4}\left\{(\vec{k} \cdot \vec{r})^{2}, \frac{p^{i}}{m}\right\}+\frac{1}{2 m}(\vec{k} \cdot \vec{r})(\vec{k} \times \vec{\sigma})^{i} \\
& -\frac{3}{4}\left\{\frac{p^{i}}{m}, \frac{r_{s}}{r}\right\}+\frac{r_{s}}{2 r} \frac{(\vec{\sigma} \times \vec{r})^{i}}{m r^{2}} \\
& -\frac{1}{2}\left\{(\mathrm{i} \vec{k} \cdot \vec{r}),\left\{\frac{p^{i}}{m}, \frac{r_{s}}{r}\right\}\right\}+\frac{3 \mathrm{i} r_{s}}{4 r} \frac{(\vec{k} \times \vec{\sigma})^{i}}{m} \\
& +\frac{1}{4}\left\{\frac{r_{s}}{r}(\mathrm{i} \vec{k} \cdot \vec{r}), \frac{p^{i}}{m}\right\} .
\end{aligned}
$$

In addition to the canonical corrections to the relativistic transition current [30, 44, 48] (kinetic corrections and magnetic coupling), this result contains a gravitational kinetic correction, and gravitational corrections to the magnetic coupling.

\section{CONCLUSIONS}

In this paper, we investigate the nonrelativistic limit of the gravitationally coupled Dirac Hamiltonian for a Dirac particle bound to the gravitational field of a planet. In order to calculate the relativistic corrections in the gravitational field, we carry out a Foldy-Wouthuysen transformation with relativistic corrections up to fourth order in the momenta and up to fourth "combined" order in the momenta and the gravitational interaction, i.e., we include terms of third order in the momenta $\left(\vec{p}^{4}\right)$ and first order in the gravitational constant $G$. Within our 
expansion, we have $|\vec{p}| \sim \xi m$, where $m$ is the fermion mass. Our calculations are thus valid up to the orders $\xi^{4}$ and $\chi \xi^{3}$, where $\chi=r_{s} / r$ is the gravitational expansion parameter. We verify that the equivalence principle holds for the gravitational interaction of particles and antiparticles, based on an inspection of the functional form of the relativistic corrections, which are all proportional to the $\beta \equiv \gamma^{0}$ matrix [see Eqs. (5) and (21)]. A coupling term proportional to $\vec{\Sigma} \cdot \vec{g}$, where $\vec{g}$ is the acceleration due to gravity, would otherwise break parity and the equivalence principle. The conceivable existence of such a term had been discussed at various places in the literature; we find that it vanishes.

One may wonder if the spin operator in our gravitational spin-orbit coupling really describes the physical spin of the electron. In order to shed light on this question, we first observe that the total angular momentum $\vec{J}=\vec{L}+\vec{S}=\vec{L}+\frac{1}{2} \vec{\Sigma}$ of the electron commutes with the free Dirac Hamiltonian (a particularly clear exposition can be found in Sec. 11.3 of Ref. [49]), as well as the Dirac-Coulomb Hamiltonian [27 29] and the DiracSchwarzschild Hamiltonian [see Eq. (24)]. One may attempt to construct a "relativistic" spin operator $\vec{Z}$ [see Eq. (22) of Ref. [50]] whose square $\vec{Z}^{2}$ "alone" (without any addition of orbital angular momentum) commutes with the fully relativistic Dirac Hamiltonian [50, 51].

In nonrelativistic limit accessed by the FoldyWouthuysen transformation, all "relativistic" spin operators discussed so far in the literature approach the limiting form $\vec{S}=\frac{1}{2} \vec{\Sigma}$ [see Eq. (16) of Ref. [50] and the text following Eq. (4) of Ref. [51]]. We can thus uniquely identify our result as the gravitational spin-orbit coupling term corresponding to the classical geodesic precession (Fokker precession) of a spinning object in the gravitational field [45], formulated within quantum mechanics and respecting the particle-antiparticle symmetry (prefactor $\beta$ ). The spin of the electron precesses due to the coupling to the gravitational field, without violating parity symmetry. We also find the gravitational analogue of the zitterbewegung term, in the Foldy-Wouthuysen transformed Dirac-Schwarschild Hamiltonian (21). The result is complemented by the gravitational corrections to the transition current (27).

\section{Acknowledgments}

The authors acknowledge helpful conversations with T. N. Moentmann, Professor M. I. Eides as well as support by the National Science Foundation (Grant PHY1068547) and by the National Institute of Standards and Technology (precision measurement grant).

\section{Appendix A: Foldy-Wouthuysen of the Free Dirac Hamiltonian}

It is instructive to carry out the Foldy-Wouthuysen transformation of the free Dirac Hamiltonian, in exactly the same two-step approach, with two iterative transformations, as described in Ref. [30] for the DiracCoulomb Hamiltonian (keeping in mind that the FoldyWouthuysen transformation of the free Dirac Hamiltonian can otherwise be carried out in a single step). The free Dirac Hamiltonian is given as,

$$
H_{\mathrm{FD}}=H_{1}=\vec{\alpha} \cdot \vec{p}+\beta m .
$$

For the first Foldy-Wouthuysen transformation, we have

$$
S_{1}=-\frac{\mathrm{i}}{2 m} \beta \mathcal{O}_{1}, \quad \mathcal{O}_{1}=\vec{\alpha} \cdot \vec{p} .
$$

The transformation is calculated as

$$
H_{2}=\mathrm{e}^{\mathrm{i} S_{1}} H_{1} \mathrm{e}^{-\mathrm{i} S_{1}}
$$

This leads to the result

$$
H_{2}=\beta\left(m+\frac{\vec{p}^{2}}{2 m}-\frac{\vec{p}^{4}}{8 m^{3}}\right)-\frac{(\vec{\alpha} \cdot \vec{p})^{3}}{3 m^{2}} .
$$

For the second Foldy-Wouthuysen transformation, we have

$$
S_{2}=-\frac{\mathrm{i}}{2 m} \beta \mathcal{O}_{2}, \quad \mathcal{O}_{2}=-\frac{(\vec{\alpha} \cdot \vec{p})^{3}}{3 m^{2}}
$$

The transformation is calculated as

$$
H_{\mathrm{FW}}=\mathrm{e}^{\mathrm{i} S_{2}} H_{2} \mathrm{e}^{-\mathrm{i} S_{2}} .
$$

The transformed Hamiltonian is

$$
H_{\mathrm{FW}}=\beta\left(m+\frac{\vec{p}^{2}}{2 m}-\frac{\vec{p}^{4}}{8 m^{3}}\right) .
$$

This result corresponds to our Eq. (21) is the limit of vanishing $r_{s}$, as it should.

All unitary transformations discussed here respect the basic symmetries of the Dirac Hamiltonian such as parity. This is essential; as an extreme example, let us briefly supplement the discussion by considering the nonrelativistic free Schrödinger Hamiltonian $H_{0}=\vec{p}^{2} /(2 m)$ and the unitary transformation $U=\exp (\mathrm{i} \vec{A} \cdot \vec{r})$, where $\vec{A}$ is a constant vector. Then, $H_{0}^{\prime}=U H_{0} U^{+}=(\vec{p}-\vec{A})^{2} /(2 m)$. Upon binomial expansion, one obtains a term proportional to $\vec{A} \cdot \vec{p}$, which breaks parity. However, the paritybreaking term in $H_{0}^{\prime}$ is an artefact generated by the parity-breaking unitary transformation. As a further illustrative remark, let us consider the the transformation $S_{1}$, given in Eq. A2 , under parity,

$$
\begin{aligned}
& S_{1}=-\frac{\mathrm{i}}{2 m} \beta \vec{\alpha} \cdot \vec{p} \\
& \quad \stackrel{\mathcal{P}}{\rightarrow} \beta\left[-\frac{\mathrm{i}}{2 m} \beta \vec{\alpha} \cdot(-\vec{p})\right] \beta=\frac{\mathrm{i}}{2 m} \vec{\alpha} \cdot \vec{p} \beta=S_{1} .
\end{aligned}
$$

By construction, the iterative Foldy-Wouthuysen transformations discussed here respect parity symmetry, and so does the final result given in Eq. (21). 
[1] R. Colella, A. W. Overhauser, and S. A. Werner, Phys. Rev. Lett. 34, 1472 (1975).

[2] U. Bonse and T. Wroblewski, Phys. Rev. Lett. 51, 1401 (1983).

[3] U. Bonse and T. Wroblewski, Phys. Rev. D 30, 1214 (1984).

[4] O. S. Ivanitskaya, Extended Lorentz transformations and their applications (in Russian) (Nauka i Technika, Minsk, USSR, 1969).

[5] O. S. Ivanitskaya, Lorentzian basis and gravitational effects in Einsteins theory of gravity (in Russian) (Nauka i Technika, Minsk, USSR, 1969).

[6] N. V. Mitskevich, Physical fields in general relativity (in Russian) (Nauka, Moscow, 1969).

[7] A. K. Gorbatsievich, Quantum mechanics in general relativity. Basic principles and elementary applications (Nauka i Technika, Minsk, 1985).

[8] O. S. Ivanitskaya, N. V. Mitskievic, and Y. S. Vladimirov, in Proceedings of the 114th Symposium of the International Astronomical Union held in Leningrad, USSR, May 1985, edited by J. Kovelevsky and V. A. Brumberg (Kluwer, Dordrecht, 1985), pp. 177-186.

[9] R. Penrose and W. Rindler, Spinors and space-time Vol. 1 (Cambridge University Press, Cambridge, UK, 1987).

[10] R. Penrose and W. Rindler, Spinors and space-time Vol. 2 (Cambridge University Press, Cambridge, UK, 1987).

[11] D. R. Brill and J. A. Wheeler, Rev. Mod. Phys. 29, 465 (1957).

[12] D. G. Boulware, Phys. Rev. D 12, 350 (1975).

[13] M. Soffel, B. Müller, and W. Greiner, J. Phys. A 10, 551 (1977).

[14] V. M. Redkov, Nonlinear Phenomena in Complex Systems 7, 250 (2011).

[15] J. Yepez, Einstein's vierbein field theory of curved space, e-print arXiv:1106.2037v1 [gr-qc].

[16] U. D. Jentschura, Phys. Rev. A 87, 032101 (2013), [Erratum Phys. Rev. A 87, 069903 (2013)].

[17] F. W. Hehl and W.-T. Ni, Phys. Rev. D 42, 2045 (1990).

[18] A. Peres, Phys. Rev. D 18, 2739 (1978).

[19] Y. N. Obukhov, Phys. Rev. Lett. 86, 192 (2001).

[20] L. L. Foldy and S. A. Wouthuysen, Phys. Rev. 78, 29 (1950).

[21] N. Nicolaevici, Phys. Rev. Lett. 89, 068902 (2002).

[22] Y. N. Obukhov, Phys. Rev. Lett. 89, 068903 (2002).

[23] A. J. Silenko and O. V. Teryaev, Phys. Rev. D 71, 064016 (2005).

[24] A. J. Silenko, Phys. Rev. A 77, 012116 (2008).

[25] Y. N. Obukhov, A. J. Silenko, and O. V. Teryaev, Phys. Rev. D 84, 024025 (2011).

[26] B. Goncalves, Y. N. Obukhov, and I. L. Shapiro, Phys. Rev. D 80, 125034 (2009).

[27] R. A. Swainson and G. W. F. Drake, J. Phys. A 24, 79 (1991).

[28] R. A. Swainson and G. W. F. Drake, J. Phys. A 24, 95 (1991).
[29] R. A. Swainson and G. W. F. Drake, J. Phys. A 24, 1801 (1991).

[30] J. D. Bjorken and S. D. Drell, Relativistic Quantum Mechanics (McGraw-Hill, New York, 1964).

[31] J. D. Bjorken and S. D. Drell, Relativistic Quantum Fields (McGraw-Hill, New York, 1965).

[32] C. Itzykson and J. B. Zuber, Quantum Field Theory (McGraw-Hill, New York, 1980).

[33] M. E. Peskin and D. V. Schroeder, An Introduction to Quantum Field Theory (Perseus, Cambridge, Massachusetts, 1995).

[34] M. Srednicki, Quantum Field Theory (Cambridge University Press, Cambridge, 2007).

[35] P. A. M. Dirac, General Relativity (Wiley, New York, NJ, 1975).

[36] R. M. Wald, General Relativity (University of Chicago Press, Chicago, IL, 1984).

[37] S. Chandrasekhar, The Mathematical Theory of Black Holes (Oxford University Press, Oxford, UK, 1992).

[38] B. Schutz, A First Course in General Relativity (Cambridge University Press, Cambridge, UK, 2009).

[39] T. Padmanabhan, Gravitation - Foundations and Frontiers (Cambridge University Press, Cambridge, UK, 2010).

[40] P. A. M. Dirac, General Theory of Relativity (Princeton University Press, Princeton, 1996).

[41] A. S. Eddington, The Mathematical Theory of Relativity (Cambridge University Press, Cambridge, England, 1924).

[42] S. Wolfram, The Mathematica Book, 4 ed. (Cambridge University Press, Cambridge, UK, 1999).

[43] T. J. Yoder and G. S. Adkins, Phys. Rev. D 86, 116005 (2012).

[44] U. D. Jentschura, Master Thesis: The Lamb Shift in Hydrogenlike Systems, in German: Theorie der LambVerschiebung in wasserstoffartigen Systemen], (University of Munich, 1996, unpublished (see e-print hep-ph/0305065)).

[45] A. D. Fokker, Kon. Akad. Weten. Amsterdam. Proc. 23, 729 (1920).

[46] I. B. Khriplovich, in Gyros, Clocks, Interferometers... : Testing Relativistic Gravity in Space (Lecture Notes in Physics 562), edited by C. Lämmerzahl, C. W. F. Everitt, and F. W. Hehl (Springer, Heidelberg, 2001), pp. 109128 .

[47] I. B. Khriplovich, Acta Phys. Polon. B Proc. Suppl. 1, 197 (2008).

[48] U. Jentschura and K. Pachucki, Phys. Rev. A 54, 1853 (1996).

[49] V. Devanathan, Quantum Mechanics (Alpha Science, Oxford, UK, 2005).

[50] L. H. Ryder, Gen. Relativ. Gravit. 31, 775 (1999).

[51] H. Bauke, S. Ahrens, C. H. Keitel, and R. Grobe, What is the relativistic spin operator?, e-print arXiv:1303.3862 1 [quantum-ph]. 